\title{
PROKLA-Redaktion
}

\section{Editorial: Währung, Sozialstaat, Arbeitsmärkte}

Europa ist ein wirtschaftlicher Riese und ein politischer Zwerg. In diesem Sinn kritisiert nicht nur der Kommissionspräsident Jacques Santer das Fehlen einer gemeinsamen europäischen $\mathrm{Au}$ Benpolitik. Während der europäischen Politik konsensfähige Handlungsziele nach außen fehlen, hat sich eine europäische 'Innenpolitik' im Laufe der Zeit durch die Rechtsprechung des Europäischen Gerichtshofs, das europaweite kommunale Wahlrecht und, nicht zuletzt, durch das Schengener Abkommen sowie seit Beginn des Jahres auch durch eine gemeinsame Währung etabliert. Doch auch hier wiederholt sich das Verhältnis eines Riesen $\mathrm{zu}$ einem Zwerg: Das monetär integrierte Europa hat bedeutend an globalem Einfluß gewonnen, während der Kompetenzrahmen für eine europäische Politik, soweit sie nicht auf die weitere Durchsetzung von Marktmechanismen zielt, weiterhin ein gnomenhaftes Dasein führt.

Die Jahrzehnte unter neoliberaler $\mathrm{He}$ gemonie hinterlassen eine europäische Architektur, in der entfesselte Kapitalinteressen gegenüber der Ausweitung staatsbürgerlicher und sozialer Rechte dominieren. Europa wird von einer Administration verwaltet, die nur sehr eingeschränkt der Kontrolle durch ein gewähltes Parlament unterliegt. Und wie der jüngste Korruptionsskandal um die Europäische Kommission vor Augen geführt hat, ist das Europäische Parla- ment bislang ein bellender Hund, der sich rasch an die Kette national geprägter Parteiloyalitäten legen läßt. Auch die Europäische Kommission verfügt freilich nur über ein beschränktes Aktionsfeld: Sie hat die unter Berücksichtigung verschiedener Interessengruppen und des Europäischen Parlaments ausgearbeiteten Vorlagen dem Ministerrat vorzulegen, damit sie gegebenenfalls in Gemeinschaftsrecht umgesetzt werden können. Und im Ministerrat werden weiterhin national vordefinierte Standpunkte ins Verhältnis gesetzt - auf kleinstem gemeinsamen Nenner. Die entscheidenden Weichenstellungen der europäischen Politik werden nicht von demokratisch legitimierten Institutionen, sondern »intergovernemental $\ll$ : auf Regierungskonferenzen vorgenommen. Auf der anderen Seite sind allerdings supranationale Strukturen entstanden, die nicht auf nationale Interessen rückgerechnet werden können. Der Europäische Gerichtshof interpretiert das europäische Vertragswerk und zwingt die Regierungen gegebenenfalls zur Umsetzung gemeinschaftlicher Bestimmungen in nationales Recht. Die zaghaften Fortschritte der europäischen Sozialgesetzgebung scheinen am ehesten auf diesem indirekten Weg zustande $\mathrm{zu}$ kommen - nachdem die Europäische Sozialcharta auf Druck der konservativen britischen Regierung zu einem bloBen Beiwerk des Maastricht-Vertrags 
herabgestuft wurde. Zahlreiche Interessengruppen haben die verstärkte Transnationalisierung der Politik verstanden und richten ihre Anliegen zunehmend ohne Umweg über nationale Parlamente gleich direkt an Brüssel.

Der erstaunlichste Schritt in Richtung transnationaler Institutionen ist sicherlich die Europäische Zentralbank. Zugleich ist es bezeichnend, daß diese Vertiefung der Integration auf eine radikale Engführung der europäischen Politik hinausläuft, und man kann vermuten, daß sie weniger von der »europäischen Idee« als vielmehr vom Druck der internationalen Märkte inspiriert ist. Die 'Vergemeinschaftung' scheint in die gleichsam entpolitisierte Sphäre des Geldes abgerutscht, dessen Verwaltung konsequenterweise noch stärker gegen politische Einflußnahme abgeschirmt ist, als es die Bundesbank je war. Für die zukünftige europäische Politik wird es maßgeblich darauf ankommen, wie weit sie sich aus der Zwangsjacke einer monomanisch auf Stabilität getrimmten Geldpolitik befreien kann. Bislang haben die »allgemeinen wirtschaftspolitischen Ziele der Gemeinschaft « - nämlich ein »umweltverträgliches Wachstum, ein hoher Grad an Konvergenz der wirtschaftlichen Leistungen, ein hohes Beschäftigungsniveau, ein hohes $\mathrm{Ma} 3$ an sozialem Schutz« etc. zu sichern keinen institutionalisierten Rückhalt erhalten, der ernsthafte Absichten zu ihrer Durchsetzung signalisieren würde. Nachdem bereits im letzten Europa-Heft der PROKLA (Heft 113: Europa I: Osteuropa und der Westen, September 1998) Elmar Altvater die Einführung des Euro im historischen Kontext von deutscher Währungsreform 1948 und Währungsunion 1990 untersucht hat, geht Klaus Müller in diesem Heft den institutionel- len Defiziten der Euro-Einführung nach. In der bisherigen Konstruktion, so seine These, läuft die gemeinsame europäische Währung auf eine riskante Konkurrenz zwischen Geld- und Wirtschaftspolitik hinaus, deren desintegrative Folgen nur durch einen Prozeß nachholender Institutionenbildung aufgefangen werden könnten.

Wenn die Einrichtung der auf eine deflationäre Geldpolitik eingeschworenen Europäischen Zentralbank unverkennbar aus Leitgedanken der Markterweiterung abgeleitet war, stellt sich die Frage, ob sich mit der europaweiten Rückkehr der Sozialdemokraten an die Macht an diesem Trend etwas ändern wird. Konservative Beobachter sehen zwar mit »Europas scharfem Linksruck Wolken über der Zukunft des Euro« aufziehen (Wall Street Journal Europe, 27.10.98), Anzeichen für eine tatsächliche Kursänderung gibt es indessen kaum: Die sozialdemokratisch dominierten Regierungen in den Mitgliedsstaaten und die von ihnen beschickten EU-Organe Rat und Kommission scheinen die alte Politik in neuem Kleid fortzuführen. Auch die neuen Regierungen nutzen den Verweis auf europäische Vorgaben, um in den einzelnen Ländern gesellschaftliche Ansprüche an die sozialstaatlichen Institutionen zurückzudrängen. Zur Erfüllung der Konvergenzkriterien wurde und wird in den Mitgliedsstaaten ein politischer Kurs eingeschlagen, dessen rigide Sparmaßnahmen nur als Sachzwänge deklariert durchsetzbar waren. Der Verweis auf europäische Direktiven ist ein willkommenes Instrument, um den Widerstand in den jeweiligen Ländern mit Verweis auf vorgeblich höhere Gründe auszuhebeln. Noch nimmt die europäische Ökonomie als unbesiegbarer, gi- 
gantischer Goliath die Arena ganz für sich in Anspruch. Dem alten Mythos nach siegt der unterlegene David - mit der Waffe des Riesen. Doch in dem von Parteien des linken Spektrums dominierten aktuellen Europa scheint es kaum eine Kraft zu geben, die auch nur versucht, der Vorherrschaft der Kapitalinteressen Grenzen zu setzen. Den Zustand und die Perspektiven der europäischen Linken nach der neoliberalen Gegenreform analysiert Frieder Otto Wolf. In der Pluralität und den Widersprüchen der Linken in Europa sieht er gleichermaßen Chancen wie Probleme für die Gewinnung einer grenzüberschreitenden Handlungsfähigkeit, die notwendig ist, um das marktradikale Muster der europäischen Integration zu korrigieren.

Daß die bislang sowohl auf einzelstaatlicher wie auf EU-Ebene vorherrschende neoliberale Politik nicht einfach auf Deregulierung reduziert werden kann, macht Petra Schaper-Rinkel in ihrem Beitrag deutlich: Die von der Europäischen Kommission forcierte Entwicklung einer »Europäischen Informationsgesellschaft« zeigt, daß die EU zum Ausbau ihrer Weltmarktposition eine Politik der Re-Regulierung betreibt, in der nationalstaatliche durch europäische Regulierungen ersetzt und zugleich politische Kräfteverhältnisse nachhaltig verschoben werden. Unter dem Primat einer europäischen Politik zur Steigerung der »Wettbewerbsfähigkeit « konnte die Kommission ihren erweiterten Handlungsspielraum in einen regulativen Rahmen umsetzen, der vornehmlich die Interessen der europäischen Unternehmen bedient. Diese re-regulierende Politik ist insofern zu Recht Anknüpfungspunkt für die sozialdemokratischen Kontinuitätsversprechen.

Trotz aller Europäisierungstendenzen verweisen politikwissenschaftliche 'Realisten' gerne darauf, daß die entscheidenden Kompetenzen nach wie vor bei den Teilnehmerstaaten verbleiben. Tatsächlich ist schwer vorstellbar, daß die sozialen Sicherungssysteme, die Steuergesetzgebung und die Außenpolitik in naher Zukunft 'vergemeinschaftet' werden. Wie unterschiedlich die nationalen Regulierungsmuster sind, zeigt der Beitrag von Heiner Ganßmann und Michael Haas anhand der Arbeitsmarktpolitik. Die konservative Kritik sieht die hohe und anhaltende Arbeitslosigkeit in Europa in einer angeblichen wohlfahrtsstaatlichen Verkrustung (der »Eurosklerose «) begründet. Tatsächlich aber haben die europäischen Länder, denen eine Reduktion der Arbeitslosigkeit gelungen ist, nicht auf einseitige Flexibilisierungsmaßnahmen gesetzt, sondern Flexibilisierung und sozialstaatliche Absicherung kombiniert. Das vielgerühmte Job-Wunder in den USA beruht dagegen auf einem Wachstum des Niedriglohnsektors, was Verarmungstendenzen Vorschub leistet und bei einer Übertragung auf europäische Verhältnisse lediglich eine starke ReHierarchisierung nach sich ziehen würde. Bereits in dem letzten Europa-Heft (PROKLA 112) wurden die Wechselwirkungen zwischen westeuropäischer Integration und osteuropäischen Transformation deutlich. Im Zuge ihrer Beitrittsgesuche sind die mittel- und osteuropäischen Länder gezwungen, sich den Bedingungen der EU im Vorhinein zu unterwerfen. Für die osteuropäischen Reformstaaten bedeutet dies, den Vorgaben der EU zu folgen, ohne einen Einfluß auf die Gestaltung der Europäischen Union und den gemeinsamen Markt zu haben (vgl. den Artikel von Peter Gowan in PROKLA 112). Dies 
gilt auch für die sozialpolitischen Reformen in Mittel- und Osteuropa. Die Herausbildung einer neuen rentenpolitischen Orthodoxie wurde von Katharina Müller bereits in PROKLA 112 untersucht, Martin Brusis analysiert nun im vorliegenden Heft den Stand der sozialstaatlichen Reformen in Polen, der Slowakei, Tschechien und Ungarn sowie die dabei zum Tragen kommenden wohlfahrtsstaatlichen Regime.

Zentral für die Entwicklungschancen der östlichen Transformationsgesellschaften sind die industriellen Beziehungen. Guy Standing gibt einen Überblick über Arbeitsmärkte und Arbeitsbeziehungen in Osteuropa und in der ehemaligen Sowjetunion. Nach Verabschiedung des realsozialistischen Vollbeschäftigungsmodells wurde angesichts steigender offener wie verdeckter Arbeitslosigkeit zwar eine aktive Arbeitsmarktpolitik eingeführt; Beschäftigte in den unsicheren Bereichen des
Arbeitsmarktes werden gleichwohl zunehmend marginalisiert. Die Entwicklungen der Arbeitsbeziehungen analysiert Standing anhand der Arbeitsgesetzgebung, der gewerkschaftliche Organisierung, der Formierung von Arbeitgeberorganisationen und tripartistischer Verhandlungsformen: Die bisherigen Regulierungsformen, so seine Diagnose, reichen nicht aus, um den Trend zur weiteren Informalisierung umzukehren und dieser dürfte längerfristig auch einen erheblichen Einfluß auf Westeuropa haben. (Mit der Informalisierung von Arbeits- und Wirtschaftsbeziehungen wird sich daher auch die im Dezember 1999 erscheinende Nummer 117 der PROKLA beschäftigen.)

Als Nachtrag zu PROKLA 113: Konzentration, Internationalisierung, Vernetzung (Dezember 1998) erscheint hier außerhalb des Schwerpunkts die Untersuchung von Thomas Haipeter über den Formwandel der Internationalisierung bei VW.

\section{Aus gegebenem Anlaß: Zum Wechsel im Abo-Versand der PROKLA}

Liebe AbonnentInnen, das 1. Heft des Jahrgangs 1999 der PROKLA erhalten Sie von der GerminalVersandbuchhandlung, (Siemensstr. 16, 35463 Fernwald) die nunmehr die Abo-Betreuung der PROKLA übernimmt.

Für die bisherige Zusammenarbeit mit der Kirschkern-Versandbuchhandlung, deren Abo-Betreuung wir seinerzeit beim Wechsel der PROKLA vom Rotbuch-Verlag zum Dampfboot übernommen haben, bedanken wir uns.

Wir sind sicher, daß die Abo-Betreuung durch die GerminalVersandbuchhandlung den von den AbonnentInnen wie vom Verlag gewünschten Standard gewährleistet. 